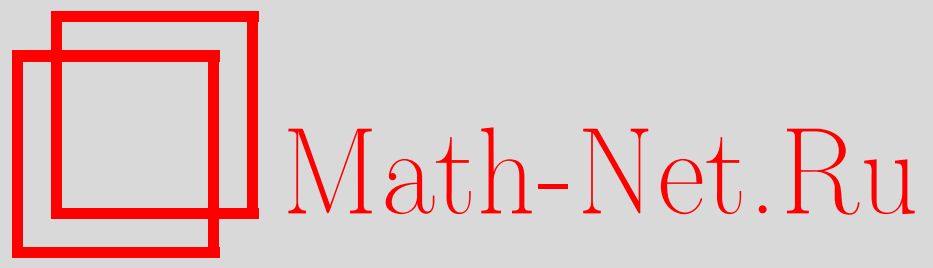

Обперос

$\Pi$.

C.

Попов,

Toпологический критерий почти-ортодополняемости любого функционала на $\ell_{2}(C(X))$, Матем. заметки, 1999, том 65, выпуск 4, 636-640

DOI: https://doi.org/10.4213/mzm1094

Использование Общероссийского математического портала Math-Net.Ru подразумевает, что вы прочитали и согласны с пользовательским соглашением http://www.mathnet.ru/rus/agreement

Параметры загрузки:

IP : 18.209 .158 .208

26 апреля 2023 г., 07:47:13 


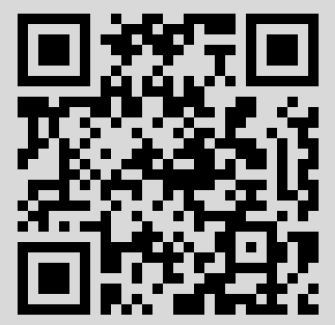




\section{ТОПОЛОГИЧЕСКИЙ КРИТЕРИЙ ПОЧТИ-ОРТОДОПОЛНЯЕМОСТИ ЛЮБОГО ФУНКЦИОНАЛА НА $\ell_{2}(C(X))$}

\section{П. С. Попов}

1. Введение. Одной из задач $K$-теории является рассмотрение свойства стягиваемости полной линейной группы операторов над $C^{*}$-гильбертовым модулем (см. [1]). Изложение теории $C^{*}$-гильбертовых модулей можно найти в [2]. Первый результат в этом направлении был получен еще в работе Диксмье и Дуади [3], где стягиваемость доказывается для группы операторов гильбертова пространства. Однако, общая теория не построена до сих пор. В работе [4] сформулированы условия (см. ниже определения 1,2$)$, достаточные для стягиваемости $\mathrm{GL}\left(\ell_{2}(\mathrm{~A})\right)$. B случае комплексного гилбертова пространства они сводятся к теореме Рисса и возможности найти для любого вектора ортогональный. В работе исследуется только коммутативный случай $\mathbb{A}=C(X)$, где $X$ - отделимый компакт. В этом случае дана переформулировка (определения 3,4 ) исходных условий, доказана их эквивалентность (теорема 1) и приведен контрпример (теорема 2). Основным результатом является критерий (теорема 4 ) выполнения этих условий - свойство типа отрицания сильной бесконечномерности. В частности, компакты, представимые в виде счетного объединения конечномерных, удоволетворяют предложенным условиям.

\section{2. Основная часть.}

ОПРЕдЕЛЕНИЕ 1. Скажем, что алгебра с единицей $\mathbb{A}$ обладает свойством $K$, если для любого функционала $v \in \mathbb{H}_{\mathbb{A}}^{\prime} \equiv \ell_{2}(\mathbb{A})^{\prime}$ и любого $\varepsilon>0$ существует вектор $h_{\varepsilon} \in \mathbb{H}_{\mathbb{A}} \equiv \ell_{2}(\mathbb{A})$ такой, что $\left|\left\langle v, h_{\varepsilon}\right\rangle\right|\left\langle\varepsilon\right.$ и $\left\langle h_{\varepsilon}, h_{\varepsilon}\right\rangle \geqslant \mathbf{1}$, где угловые скобки означают как скалярное произведение в $\mathbb{H}_{\mathbb{A}}$, так и применение функционала к вектору.

ОПРЕДЕЛЕНИЕ 2. Скажем, что $\mathbb{A}$ обладает свойством $E$, если для любого функционала $v=\left(v_{1}, \ldots, v_{n}, \ldots\right) \in \mathbb{H}_{\mathbb{A}}^{\prime}$ и любого $\varepsilon>0$ можно найти функционал $g=\left(g_{1}, \ldots, g_{n}, \ldots\right) \in \mathbb{H}_{\mathbb{A}}^{\prime}$ и число $k \in \mathbb{Z}$ такие, что

$$
\|f-g\|<\varepsilon, \quad f_{i}=g_{i}, \quad i=k+1, k+2, \ldots,
$$

и $\left.g\right|_{L_{k}}: L_{k} \rightarrow A$ является эпиморфизмом, где $L_{n}=\left\{\left(a_{1}, \ldots, a_{n}, 0,0, \ldots\right)\right\}$.

Следующие два утверждения дают топологическую характеризацию модулей $\mathbb{H}_{\mathbb{A}}$ и $\mathbb{H}_{\mathbb{A}}^{\prime}$ (доказательство см. в [5]). Всюду далее $\mathbb{A} \equiv C(X)$, где $X$ является отделимым компактом.

ПРЕДЛОЖЕнИЕ 1. Пространство $C\left(X,\left(\ell_{2}(\mathbb{C})\right.\right.$, strong $\left.)\right)$ - множество всех непрерывных отобрахсений из хаусдорфова компакта в гильбертово комплексное пространство с топологией нормы, - будучи снабжено $C(X)$-линейным скалярным произведением $\langle v, u\rangle(x)=\langle v(x), u(x)\rangle_{\ell_{2}}$, изоморфно модулю $\mathbb{H}_{\mathbb{A}}$.

Работа выполнена при поддержке фонда INTAS, грант № 96-1099. 
ПРЕДЛОЖЕНИЕ 2. Пространство $C\left(X,\left(\ell_{2}(\mathbb{C})\right.\right.$, weak $\left.)\right)$ - множсество всех непрерывных отобрахений из хаусдорфова компакта в комплексное аильбертово пространство со слабой топологией (иньми словами, множество всех покоординатно непрерывных и равномерно ограниченных по норме отображсний), - изоморфно модулю $\mathbb{H}_{\mathbb{A}}^{\prime}$. При фиксированном изоморфизме из предложсения 1 действие $v \in \mathbb{H}_{\mathbb{A}}^{\prime}$ на $u \in \mathbb{H}_{\mathbb{A}}$ задается формулой $\langle v, u\rangle(x)=\langle v(x), u(x)\rangle_{\ell_{2}}$.

Теперь мы можем переформулировать свойства $K, E$ для коммутативного случая. Предварительно введем пространство $\mathbb{H}_{w} \subset \mathbb{H}_{\mathbb{A}} \subset \mathbb{H}_{\mathbb{A}}^{\prime}$. Определим егокак $\mathbb{H}_{w}=\left\{v \in \mathbb{H}_{\mathbb{A}}: P_{n}(v(x)) \equiv v(x)\right.$ для некоторого $n\}$, где $P_{n}$ - проектор в $\ell_{2}$ на первые $n$ базисных векторов. Кроме того, нам потребуется следующая лемма.

ЛЕмма 1. Пусть $u \in \mathbb{H}_{\mathbb{A}}^{\prime}$, причем $\|u(x)\|>\beta$ для любого $x \in X$. Тогда для любой константы $\alpha<\beta$ существует число $n$ такое, что $\left\|P_{n} u(x)\right\|>\alpha$ всюду на компакте.

ДоказАтельство. Рассмотрим $X_{i}=\left\{x \in X:\left\|P_{n} u(x)\right\|>\alpha\right\}$. Эти множества открыты, вложены и образуют покрытие, так как для любого $x$ существует число $n$ такое, что $\left\|P_{n}(u(x))\right\|>\alpha$. Выберем конечное подпокрытие, максимальный номер элементов подпокрытия даст нам число $n$.

ОПРЕДЕЛЕНИЕ 3 . Компакт $X$ обладает свойством $K$, если для любого функционала $v \in \mathbb{H}_{\mathbb{A}}^{\prime}$ и любого $\varepsilon>0$ существует элемент $h_{\varepsilon} \in \mathbb{H}_{\mathbb{A}}$ такой, что всюду на $X$ выполнено

$$
\left\|h_{\varepsilon}(x)\right\| \geqslant 1, \quad\left|\left\langle h_{\varepsilon}(x), v(x)\right\rangle\right|<\varepsilon .
$$

Заметим, что вместо условия $h_{\varepsilon} \in \mathbb{H}_{\mathbb{A}}$ мы можем потребовать формально более сильное $h_{\varepsilon} \in \mathbb{H}_{w}$ или формально более слабое $h_{\varepsilon} \in \mathbb{H}_{\mathbb{A}}^{\prime}$. Однако, все три условия оказываются эквивалентными: достаточно к $h_{\varepsilon / 2}$ применить результат леммы 1 с константой $\alpha=1 / 2$. Кроме того, нормировкой можно добиться, чтобы $\left\|h_{\varepsilon}(x)\right\| \equiv 1$.

ОПРЕДЕЛЕНИЕ 4 . Компакт $X$ обладает свойством $E$, если для любого функционала $v \in \mathbb{H}_{\mathbb{A}}^{\prime}$ и любого $\varepsilon>0$ существуют элемент $v_{\varepsilon} \in \mathbb{H}_{\mathbb{A}}$ и число $n \in \mathbb{Z}$ такие, что всюду на $X$ выполнено

$$
\left\|v_{\varepsilon}(x)-v(x)\right\| \leqslant \varepsilon, \quad\left(1-P_{n}\right)\left(v_{\varepsilon}(x)-v(x)\right)=0, \quad P_{n} v_{\varepsilon}(x) \neq 0 .
$$

Заметим, что $E$ не зависит от выбора в качестве основного для $\ell_{2}$ поля $\mathbb{C}$ или $\mathbb{R}$, так как отображение $\Theta: \ell_{2}(\mathbb{C}) \rightarrow \ell_{2}(\mathbb{R})$, определенное как $\Theta\left(x_{1}, \ldots, x_{n}, \ldots\right)=\left(\operatorname{Im} x_{1}, \operatorname{Re} x_{1}, \ldots, \operatorname{Im} x_{n}\right.$, $\left.\operatorname{Re} x_{n}, \ldots\right)$, сохраняет норму и слабую топологию.

Tеорема 1. Свойства $E$ и $К$ әквивалентны.

ДокАЗАТЕльство. Докажем импликацию $K \Longrightarrow E$. Пусть для данных $v$ и $\varepsilon / 2$ выполнено свойство $K$ : всюду на компакте $\left\|h_{\varepsilon / 2}(x)\right\|=1$ и $\left|\left\langle h_{\varepsilon / 2}(x), v(x)\right\rangle\right| \leqslant \varepsilon / 2$. Как было замечено, можно считать, что существует число $n$ такое, что $P_{n} h_{\varepsilon / 2}(x) \equiv h_{\varepsilon / 2}(x)$. Положим $v_{\varepsilon}(x):=v(x)+$ $\varepsilon h_{\varepsilon / 2}(x)$. Тогда всюду на компакте $\left\|v_{\varepsilon}(x)-v(x)\right\|=\varepsilon$ и $P_{n}\left(v_{\varepsilon}(x)-v(x)\right)=$ $v_{\varepsilon}(x)-v(x)$. Рассмотрим скалярное произведение

$$
\left|\left\langle P_{n} v_{\varepsilon}(x), h_{\varepsilon / 2}(x)\right\rangle\right|=\left|\left\langle v_{\varepsilon}(x), h_{\varepsilon / 2}(x)\right\rangle\right|=\left|\left\langle v(x), h_{\varepsilon / 2}(x)\right\rangle+\varepsilon\left\|h_{\varepsilon / 2}\right\|^{2}\right|>\frac{\varepsilon}{2} .
$$

Скалярное произведение отлично от 0 всюду на компакте. Следовательно, отображение $P_{n} v_{\varepsilon}(x)$ отлично от 0 для любого $x \in X$.

Докажем импликацию $E \Longrightarrow K$. Пусть для данных $v$ и $\varepsilon / 2$ выполнено свойство $E$ : всюду на компакте

$$
\left\|v_{\varepsilon}(x)-v(x)\right\| \leqslant \varepsilon, \quad\left(1-P_{n}\right)\left(v_{\varepsilon}(x)-v(x)\right)=0, \quad P_{n} v_{\varepsilon}(x) \neq 0 .
$$

Рассмотрим вектор $P_{2 n} v_{\varepsilon}(x)=\left(g_{1}(x), g_{2}(x), \ldots, g_{2 n-1}(x), g_{2 n}(x), 0, \ldots\right) \neq 0$. Положим

$$
h_{\varepsilon}(x):=\frac{\left(-g_{2}^{*}(x), g_{1}^{*}(x), \ldots,-g_{2 n}^{*}(x), g_{2 n-1}^{*}(x), 0, \ldots\right)}{\left\|P_{2 n} v_{\varepsilon}(x)\right\|},
$$


где норма в знаменателе непрерывна и отлична от 0 . Тогда $\left\|h_{\varepsilon}(x)\right\|=1$ для любого $x \in X$ и, поскольку $\left\langle h_{\varepsilon}(x), v_{\varepsilon}(x)\right\rangle \equiv 0$,

$$
\left|\left\langle h_{\varepsilon}(x), v(x)\right\rangle\right| \leqslant\left|\left\langle h_{\varepsilon}(x), v(x)-v_{\varepsilon}(x)\right\rangle\right|+\left|\left\langle h_{\varepsilon}(x), v_{\varepsilon}(x)\right\rangle\right| \leqslant\left\|h_{\varepsilon}(x)\right\| \cdot\left\|v_{\varepsilon}(x)-v(x)\right\|+0 \leqslant \varepsilon .
$$

Заметим, что последнее доказательств годится как в комплексном, так и в действительном случае, т.е. $K$ также не зависит от выбора в качестве основного для $\ell_{2}$ поля $\mathbb{C}$ или $\mathbb{R}$. Этим фактом мы будем пользоваться при доказательстве теоремы о "плохом компакте". Теорема доказана.

Пусть далее $B^{\infty} \equiv\left(B(0,1) \in \ell_{2}(\mathbb{R})\right.$, weak $)$ - шар в $\ell_{2}(\mathbb{R})$, снабженный слабой топологией. В следующей теореме доказано, что $C\left(B^{\infty}\right)$ не удовлетворяет свойствам $K, E$. Заметим, что это следует из теоремы 4 (см. ниже) и теорем Брауэра, однако будет представлено доказательство несколько более сильного факта.

Teоpema 2. Пусть $X \equiv B^{\infty}, v=\left.\operatorname{Id}\right|_{B^{\infty}}$. Тогда свойство $K$ не выполнено для $v$. Более того, для любого $\varepsilon<1$ и любого $h \in \mathbb{H}_{\mathbb{A}}^{\prime}$, всюду большего по норме 1 , существует точка $x^{0} \in B^{\infty}$ такая, что $\left|\left\langle h\left(x^{0}\right), x^{0}\right\rangle\right|>\varepsilon$.

ДокАЗАтЕльство. Предположим противное. Пусть для $\varepsilon<1$ существует $h_{\varepsilon} \in \mathbb{H}_{\mathbb{A}}^{\prime}$ такой, что $\left|\left\langle h_{\varepsilon}(x), x\right\rangle\right|<\varepsilon$ и $\left\|h_{\varepsilon}(x)\right\|>1$ для любого $x \in B^{\infty}$. Тогда по лемме 1 существует число $n$ такое, что $P_{2 n+1} h(x)>\varepsilon$ всюду на $B^{\infty}$. Рассмотрим сферу $S^{2 n}$, вложенную в $\operatorname{Span}\left(e_{1}, \ldots, e_{2 n+1}\right)$. Тогда для любого $y \in S^{2 n}$ вектор $P_{2 n+1} h_{\varepsilon}(y)$ не параллелен $y$, ибо если они параллельны в точке $y_{0}$, то

$$
\varepsilon>\left|\left\langle P_{2 n+1} h_{\varepsilon}\left(y_{0}\right), y_{0}\right\rangle\right|=\left\|y_{0}\right\| \cdot\left\|P_{2 n+1} h_{\varepsilon}(x)\right\|>\varepsilon .
$$

Проекция $P_{2 n+1} h_{\varepsilon}(x)$ на $T_{x} S^{2 n}$ - непрерьвное поле, не обращающееся в 0. Получили противоречие. Теорема доказана.

Теорема 3. Пусть $X=\bigcup_{i=0}^{\infty} X_{i}, X_{i}$ обладает свойствами $K, E$. Тогда $X$ обладает свойствами $K, E$.

ДоКАЗАтЕльство. Разобьем $\ell_{2}$ в прямую сумму гильбертовых пространств $\ell_{2}=\bigoplus_{i=0}^{\infty} \ell_{2}^{i}$ и $v(x)=\bigoplus_{i=0}^{\infty} v^{i}(x)$. Так как $X_{i}$ обладает свойством $K$, существует отображение $h_{\varepsilon / 2^{i+4}}^{i}: X_{i} \rightarrow \ell_{2}^{i}$ такое, что для любого $x \in X_{i}$ вьполнено

$$
\left\|h_{\varepsilon / 2^{i+4}}^{i}(x)\right\|>1, \quad\left|\left\langle h_{\varepsilon / 2^{i+4}}^{i}(x), v^{i}(x)\right\rangle\right| \leqslant \frac{\varepsilon}{2^{i+4}}, \quad P_{n_{i}} h_{\varepsilon / 2^{i+4}}^{i}(x)=h_{\varepsilon / 2^{i+4}}^{i}(x) .
$$

Продолжим $n_{i}$ координатных функций на весь компакт $X$. Тогда существует окрестность $W\left(X_{i}\right)$ такая, что $\left\|h_{\varepsilon / 2^{i+4}}^{i}(x)\right\|>1 / 2$ и $\left|\left\langle h_{\varepsilon / 2^{i+4}}^{i}(x), v^{i}(x)\right\rangle\right| \leqslant \varepsilon / 2^{i+3}$. Рассмотрим окрестность $V\left(X_{i}\right)$, содержащую $X_{i}$ и содержащуюся в $W\left(X_{i}\right)$, и функцию $\tau(x)$ такую, что $\operatorname{Supp}(\tau) \in W\left(X_{i}\right)$, $\left.\tau(x)\right|_{V\left(X_{i}\right)}=1$. Тогда для $h^{i}(x):=2 \tau(x) h_{\varepsilon / 2^{i+4}}^{i}(x)$ выполнено

$$
\left|\left\langle h^{i}(x), v^{i}(x)\right\rangle\right|<\varepsilon / 2^{i+2} \text { всюду на } X, \quad\left\|h^{i}(x)\right\|>1 \quad \text { для всех } x \in V\left(X_{i}\right) .
$$

Выберем из $V\left(X_{i}\right)$ конечное подпокрытие $X=U_{j \in \psi} V\left(X_{j}\right)$. Введем функцию

$$
h_{\varepsilon}(x)=\bigoplus_{j \in \psi} h^{j}(x)
$$

Легко видеть, что эта функция всюду больше по норме 1 (так как $V\left(X_{j}\right), j \in \psi$, образуют покрытие), и выполнено неравенство

$$
\left|\left\langle v(x), h^{i}(x)\right\rangle\right| \leqslant \varepsilon \sum_{j \in \psi} \frac{1}{2^{j+2}}<\varepsilon .
$$

Теорема доказана. 
3. $\ell_{2}$-несущественные отображения. Скажем, что отображение $f: X \rightarrow B^{n}=$ $B(0,1) \in \mathbb{R}^{n} n$-существенно, если для любого отображения $g: X \rightarrow B^{n}$ такого, что $f(x)=g(x)$ для всех $x \in f^{-1}\left(\delta B^{n}=S^{n-1}\right)$, точка $O=(0, \ldots, 0)$ лежит в образе $g$. Как показано в [6], требование $n$-несущественности любого отображения эквивалентно неравенству $\operatorname{Dim} X<n$.

ОПредЕЛЕниЕ 5. Отображение $f: K \rightarrow B^{\infty}=\left(B(0,1) \in \ell_{2}\right.$, weak) называется $\ell_{2}$-существенныл, если для любого $n$ отображение $P_{n} f \rightarrow B^{n} n$-существенно. Компакт называется $\ell_{2}$-несущественным, если любое его отображение в $B^{\infty}$ не является $\ell_{2}$-существенным. Заметим, что конечномерные компакты удоволетворяют этому свойству.

Предложенное выше свойство сходно со свойством существенности отображения в гильбертов кирпич ( $B^{\infty}$ в данном вьше определении заменяется на гильбертов кирпич). Это свойство (см. [6]) играет важную роль в теории бесконечномерных пространств. Вопрос о взаимосвязи этих двух определений открыт. Нашей целью является доказательство эквивалентности определения 5 свойствам $K, E$. Откуда следует выполнение этих свойств как для конечномерных компактов, так и (применяя теорему 3) для компактов, представимых в виде объединения таковых.

Лемма 2. Существует ретракиия Ret: $\ell_{2} \rightarrow B^{\infty}$ непрерывная в слабой топологии.

ДокАЗАТЕЛЬСтво. Построение. Определим функцию $t(x, y): \mathbb{R}^{2} \rightarrow \mathbb{R}$ следующим образом:

$$
t(x, y):= \begin{cases}y, & \text { если } x^{2}+y^{2} \leqslant 1 \\ \operatorname{sgn}(y) \sqrt{1-x^{2}}, & \text { если } x^{2} \leqslant 1 \text { и } x^{2}+y^{2} \geqslant 1, \\ 0, & \text { если } x^{2} \geqslant 1 .\end{cases}
$$

Легко видеть, что функция $t(x, y)$ непрерьвна. Определим $\operatorname{Ret}(\gamma)$ на $\gamma=\left(\gamma_{1}, \ldots, \gamma_{i}, \ldots\right)$ :

$$
\begin{aligned}
\operatorname{Ret}(\gamma)_{1} & :=t\left(0, \gamma_{1}\right), \quad \operatorname{Ret}(\gamma)_{2}:=t\left(\left|\gamma_{1}\right|, \gamma_{2}\right)=t\left(\left\|P_{1} \gamma\right\|, \gamma_{2}\right), \ldots \\
\operatorname{Ret}(\gamma)_{n} & :=t\left(\left\|P_{n-1} \gamma\right\|, \gamma_{n}\right), \ldots
\end{aligned}
$$

Для векторов $\gamma$ из $\ell_{2}$ по норме $\leqslant 1$ эта функция тождественная. Рассмотрим $\operatorname{Ret}(\gamma)$, где $\|\gamma\|>1$. Пусть $n_{\min }=\min \left(n:\left\|P_{n} \gamma\right\|>1\right)$. Тогда утверждается, что $\operatorname{Ret}(\gamma)$ представима в виде

$$
\operatorname{Ret}(\gamma)=P_{n_{\min }-1} \gamma+e_{n_{\min }} \operatorname{sgn}\left(\gamma_{n_{\min }}\right) \sqrt{1-\left\|P_{n_{\min }-1}\right\|^{2}}
$$

Легко видеть, что $\|\operatorname{Ret}(\gamma)\|=1$. Функция Ret является ретракцией в теоретико-множественном смысле. Нам необходимо доказать непрерьвность. Она следует из ограниченности по норме и покоординатной непрерьвности, которая вытекает из непрерьвности $t$ и $\left\|P_{n}(\gamma)\right\|$. Построение завершено.

Теорема 4. Свойства $K, E$ и $\ell_{2}$-несущественности эквивалентны.

ДокаЗАТЕЛЬство. Докажем, что из $\ell_{2}$-несущественности следует свойство $E$. Фиксируем $v: X \rightarrow \ell_{2}$ и $\varepsilon>0$. Отображение $f_{\varepsilon}=\operatorname{Ret}(v / \varepsilon)$ несущественно. Существуют целое $n$ и отображение $g: X \rightarrow B^{n}$ такие, что $g(x)=P_{n} f_{\varepsilon}(x)$ для всех $x \in\left(P_{n} f_{\varepsilon}\right)^{-1}\left(S^{n-1}\right)$ и всюду $g(x) \neq 0$. Положим $v_{\varepsilon}(x):=\varepsilon\left(g(x)-P_{n} f_{\varepsilon}(x)\right)+v(x)$. Очевидно, что $P_{n}\left(v_{\varepsilon}-v\right) \equiv 0$ и, кроме того, для любого $x \in X$ выполнено

$$
\left\|v(x)-v_{\varepsilon}(x)\right\|=\varepsilon\left\|g(x)-P_{n} f_{\varepsilon}(x)\right\| \leqslant 2 \varepsilon .
$$

Для выполнения свойства $E$ с константой $2 \varepsilon$ необходимо доказать, что $P_{n} v_{\varepsilon}(x) \neq 0$. Рассмотрим два случая.

Если $\left\|P_{n} v(y)\right\| \geqslant \varepsilon$, то, как следует из построения функции Ret, выполнено $\left\|P_{n} f_{\varepsilon}(y)\right\|=1$. Значит, $g(y)=P_{n} f_{\varepsilon}(y)$. Тогда $P_{n} v_{\varepsilon}(y)=P_{n} v(y) \neq 0$.

Если же $\left\|P_{n} v(y)\right\|<\varepsilon$, то $P_{n} v_{\varepsilon}(y)=\varepsilon g(x)+P_{n} v(x)-\varepsilon P_{n} \operatorname{Ret}(v(x) / \varepsilon)=\varepsilon g(x) \neq 0$, что и требовалось доказать. 
Докажем, что из свойства $E$ следует $\ell_{2}$-несущественность. Для фиксированного $v: X \rightarrow B^{\infty}$ и $\varepsilon=1 / 7$ существует отображение $h: X \rightarrow B^{\infty}$ такое, что $\|h(x)\|=1,|\langle h(x), v(x)\rangle| \leqslant 1 / 7$ и $P_{n} h(x)=h(x)$ всюду на $X$.

Рассмотрим непрерьвную функцию $\tau: \mathbb{R} \rightarrow \mathbb{R}$ такую, что

$$
\operatorname{Supp}(\tau) \in[-2 / 3,2 / 3],\left.\quad \tau\right|_{[-1 / 3,1 / 3]}=1 .
$$

Утверждается, что отображение

$$
g(x):=P_{n} v(x)+\frac{1}{6} \tau\left(\left\|P_{n} v(x)\right\|\right) h(x)
$$

обеспечивает несущественность $v(x)$ в размерности $n$. Действительно, $g(x)$ совпадает с $P_{n} v(x)$ на $\left\{x:\left\|P_{n} v(x)\right\|=1\right\}$ и $g(x)$, как будет доказано ниже, не принимает значение 0 . Рассмотрим два случая.

Если $\left\|P_{n} v(x)\right\| \geqslant 1 / 3$, то $\|g(x)\| \geqslant 1 / 3-1 / 6>0$.

Если $\left\|P_{n} v(x)\right\| \leqslant 1 / 3$, то $g(x) \neq 0$. Поскольку если существует точка $x_{0}$ такая, что $g\left(x_{0}\right)=0$, то $P_{n} v\left(x_{0}\right)=-h\left(x_{0}\right) / 6$. Умножим скалярно обе части равенства на $h\left(x_{0}\right)$, получим противоречие: $1 / 7 \geqslant\left|\left\langle h\left(x_{0}\right), v\left(x_{0}\right)\right\rangle\right|=1 / 6$. Итак, отображение $f$ является $\ell_{2}$-несущественным. Теорема доказана.

Автор выражает искреннюю благодарность В. М. Мануйлову, А. С. Мищенко и Е. В. Троицкому за полезное обсуждение результатов работы.

\section{СПИСОК ЦИТИРОВАННОЙ ЛИТЕРАТУРЫ}

1. Троицкий Е. В. // Функцион. анализ и его прилож. 1986. Т. 20. №4. С. 58-64. 2. Lance E. C. Hilbert $C^{*}$-Modules - a Toolkit for Operator Algebraists. London Math. Soc. Lecture Note Ser. V. 210. Cambridge: Univ. Press, 1995. 3. Dixmier J., Douady A. // Bull. Soc. Math. France. 1963. V. 91. Р. 227-284. 4. Троицкий Е. В. // Труды международной конференции "Функциональные пространства. Дифференциальные операторы. Проблемы математического образования", посвященной 75-летию члена-корр. РАН профессора Л. Д. Кудрявцева. Москва, 1998. С. 63. 5. Frank M. // Ann. Global Anal. Geom. 1985. V. 3. № 2. Р. 155-171. 6. Александров П. С., Пасынков Б.А. Введение в теорию размерностей. Введение в теорию топологических пространств и общую теорию размерностей. М.: Наука, 1973. 